\title{
Robust Tracking Control of Multijoint Robots based on Adaptive Fuzzy Observer
}

\author{
Na Wang ${ }^{1}$, Miao Dang ${ }^{1}$, Jimeng Zhang, ${ }^{1, *}$ and Junzhe $\mathrm{Hu}^{2}$ \\ ${ }^{1}$ Henan Polytechnic Institute, Nanyang Henan 473000, China \\ ${ }^{2}$ Kaiserslautern Technical University, Kaiserslautern Rhineland-Pfalz 67663, Germany
}

Received 3 June 2020; Accepted 11 August 2020

\begin{abstract}
Multijoint robot is a complex and variable electromechanical coupling system, and the inherent parameters of its model, friction, and external disturbance have important effects on the performance of the control system. A robust control method of multijoint robots based on an adaptive fuzzy observer was proposed in this study to improve the influence of uncertain parameters and external disturbances on the control performance effectively. First, this study constructed the error dynamic model of the system according to the mechanical characteristics of the multijoint robot system. Second, the adaptive fuzzy estimation and compensation of the robot total disturbance were conducted by using fuzzy theory, and the $L_{2}$ gain robust controller was designed. Finally, the stability of the system was analyzed by the Hamiltonian-Jacobi inequality theorem, and the effectiveness of the control method was verified by simulation. Results show that when model parameter perturbation and external disturbance simultaneously exist in the system, the maximum angular displacement error and the maximum angular velocity tracking error of the robot are reduced by 20 times and 9.57 times, respectively, compared with the sliding mode control method. The observation accuracy of the designed adaptive fuzzy observer is four times higher than that of the conventional fuzzy observer, the number of fuzzy rules is reduced, and the tracking speed of the system is improved. The output torque of the proposed control method has a small peak torque, which is reduced by six times compared with the sliding mode control, and the control curve is smooth. The study provide a certain basis for the multijoint robot to adapt to the complex and changeable environment.
\end{abstract}

Keywords: Multidegree of freedom robot, Adaptive fuzzy observer, Robust control

\section{Introduction}

Robot, an important executive unit in intelligent manufacturing production lines, is widely used in mechanical processing, automobile manufacturing, assembly and logistics palletizing, and other fields. Especially in the assembly field, the robot has high requirements for dynamic performance, such as work efficiency, response speed, trajectory tracking accuracy, and stability time, which provides a strong guarantee for production capacity increase, quality improvement, and cost reduction [1-6]. Industrial robot, an automatic device that integrates computer, automation, and information technologies, is an important support for the gradual development of product design and production toward automation and informatization. However, with the complexity of the working environment and the increasing difficulty of working conditions, the robot system has many uncertain factors, such as unmodeled dynamics, parameter measurement error, load change, external disturbance, and friction between joints, which will seriously affect the control performance of the system. Moreover, meeting the requirements of high precision, speed, and performance is difficult. Therefore, the highprecision tracking and control of robots with uncertainties, such as modeling errors and external disturbances, have crucial practical significance.

Robots are developing toward high precision, speed, and

*E-mail address: mshf422@163.com

ISSN: 1791-2377 @ 2020 School of Science, IHU. All rights reserved. doi:10.25103/jestr.134.15 multidegree of freedom (DOF). For high-precision occasions, robots with a compensation function can meet the needs of aerospace high-precision operation equipment processing [4]. Robots based on lidar can realize accurate whole making and improve the accuracy, flexibility, and real-time performance of drilling systems [5]. Considering high space and weight requirements, such as oil-immersed transformer, highprecision inspection robots can realize the precise observation of a specific point in the depth direction [6]. The multi-DOF robot for heavy-duty friction stir welding can realize the dexterous welding of high-strength large-scale complex curved surface parts in the working space and heavy load conditions [7]. The 6-DOF industrial robot equipped with a laser tracker linkage closed-loop control can complete high-precision assembly of satellite components [8]. With the change in the working environments of robots and the complexity of working conditions, the uncertainty of robot model parameters and external interference has a serious impact on the performance of the system and cannot be used normally. Therefore, the realization of highprecision control with strong robustness and antiinterference capability is obtained in this study based on the corresponding control methods.

Therefore, according to the structural characteristics of industrial robot systems, this study establishes a complex dynamic model with model parameter uncertainty and external disturbance and conducts the disturbance observation using an adaptive fuzzy observer. Then, a robust adaptive controller is designed on the basis of $L_{2}$ gain robust control theory, and the adaptive fuzzy observer is employed to observe and compensate for the total disturbance of the 
system. The robust control method of the designed high performance provides the basis for the application of highprecision robot tracking in complex and changeable environments.

\section{State of the art}

At present, scholars have conducted numerous studies on robot control, including classical control model, adaptive control based on the online estimation of system model parameters, sliding mode control with disturbance suppression, and intelligent control. The controller comprising proportional integral differential (PID) control integrates the past, present, and future information of error and has a simple structure. Thus, the controller is still considered to be the mainstream control mode in industrial robots. Huynh et al. [9] designed a force/position hybrid control method of a double closed-loop PID controller for a 6-DOF parallel robot. The impedance control model was adopted to ensure safe and accurate interaction when contacting the surface of the object, eliminate the position error caused by the dynamic coupling effect between the manipulator and the mechanical system, and realize the safety and precise control of the robot when contacting the object surface. The proposed method had high requirements for the environment, and the system performance was reduced when the system model parameters remarkably changed. Ma et al. [10] proposed a nonlinear PID controller to solve the problems of overshoot and large amplitude fluctuation in the traditional PID control of autonomous underwater vehicles in deep motion. The control gain of the proposed method was fixed and had a good tracking effect when the model parameters were fixed and the external disturbance was small. Zheng et al. [11] designed a fuzzy PID tracking controller, which applied fuzzy rules to adjust the proportion and differential gain on-line, to enhance adaptability of PID control to the external environment changes. The structure of the controller was complex, and determining the fuzzy rules was difficult. However, the adopted method enhanced the external interference suppression capability to a certain extent. Resonance point was observed in the joint servo control system of industrial robots due to the flexibility of the reducer, and the traditional proportional-integral control vibrated under extreme position command. Following the internal model control principle, Huang et al. [12] designed a new robot vibration suppression method based on filters to enhance the robustness of the system. The proposed method was sensitive to the changes in model parameters and had limited capability to suppress external disturbances. Jiao et al. [13] combined adaptive and internal model controls to improve the model dependence of the internal model control; however, the internal model control was sensitive to external disturbances. Liu et al. [14] designed a time-delay observer to observe and compensate for the disturbance to overcome the influence of external disturbance on system performance and improve the dependence on the system model. However, the observer elicited the high-frequency signal of the system. Thus, low-pass filtering was conducted to complicate control. Considering the parameter uncertainty of flexible joint robot systems, Luc et al. [15] designed a robust adaptive control method based on cascade structure. The proposed method ensured the high-precision trajectory tracking of the robot. However, determining the system structure was required, and the unknown parameters met the linear requirements. Baek et al. [16] used the time-delay observer to estimate and compensate for the complex nonlinear dynamics and disturbance of the robot to improve the tracking accuracy of the system. However, low-pass filtering was still needed in time-delay estimation. Sliding mode control was an effective control method for uncertain robot systems because of its strong robustness to parameter and external disturbances. Rubio [17] applied the proposed method to the control of robot systems, and the nonlinear feedback control realized the deterministic partial feedback control. The switching robust control was employed to compensate for the uncertainties and external disturbances of the model. The proposed method required the satisfaction of the matching conditions by the model parameters and revealed the inherent chattering phenomenon of the sliding mode control. Beak et al. [18] applied an adaptive sliding-mode position control method for robots combining the adaptive control method and sliding mode control to improve the buffeting problem. This applied method enhanced the adaptive and chattering suppression capabilities near the sliding manifold. However, the proposed method still failed to eliminate chattering. Nair et al. [19] designed an adaptive terminal sliding mode control. In this design, the control method maximized the strong capability of the terminal attractor to suppress the system interference and adjusted the control gain on-line. Moreover, the fractional calculus operator was used in the proposed method, and its construction was complex in practice, which limited its application. Worthmann et al. [20] applied predictive control to nonholonomic mobile robot systems. Although the applied method was robust to uncertainties, it was sensitive to the changes in system parameters based on the model parameters of the system. Incremona et al. [21] combined predictive and sliding mode controls to overcome the influence of sensitivity to the change in system parameters. However, the control method increased the complexity of the system controller, thus complicating the identification of the control parameters. Wang et al. [22] applied the linear extended state observer to estimate the friction and applied it to the friction compensation in the nonlinear controller. The linear observer had a good convergence rate when the system state was far away from the equilibrium point. However, the convergence speed was slow when the system state approached the equilibrium point. Second-order feedforward external force, nonlinear disturbance, and convolution torque observers were also applied for robot disturbance estimation, but these methods were highly dependent on model parameters [23-25]. Neural network fuzzy theory had the characteristics of arbitrary nonlinearity approximation and was also applied for nonlinear system interference estimation and compensation [26-28]. In the application, the structure of the neural network and fuzzy controls was relatively complex, and determining the weight and structure of the network was difficult. Moreover, the unified form of fuzzy control rules, which were difficult to apply, were not found.

The above study results mainly focused on the influence of system model parameter uncertainty. However, the study on system friction torque, load disturbance, and model parameter uncertainty was limited. The present study established an error dynamic model with disturbance and uncertain parameters. The fuzzy observer was employed to observe and compensate for the total disturbance of the system, and a high-performance robot control method was designed to meet the requirements of working in complex and changeable environments. 
The remainder of this study is organized as follows. Section 3 constructs the uncertain mathematical model of the robot, designs the robust adaptive control method based on fuzzy observation compensation, and conducts the stability analysis. Section 4 verifies the effectiveness of the proposed control method by comparison with the traditional control method. Section 5 provides conclusions.

\section{Methodology}

\subsection{System description}

Fig. 1 shows a typical two joint robot.

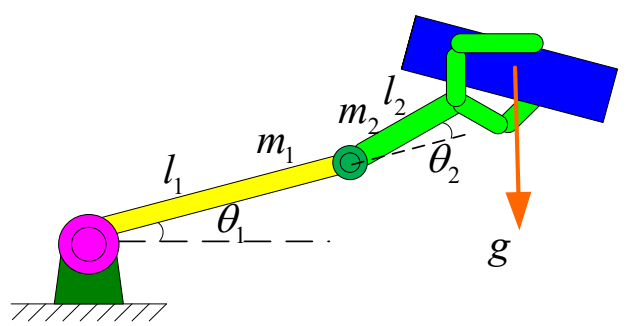

Fig. 1. Typical two-joint robot

The dynamic equation of $n$-joint multi-DOF robot can be expressed as follows: [9]

$$
M_{0}(q) \ddot{\theta}+G_{0}(\theta, \dot{\theta}) \dot{\theta}+V_{0}(\theta)+h(\theta, \dot{\theta})+d=\tau
$$

where $\theta \in R^{n}, \dot{\theta} \in R^{n}$, and $\ddot{\theta} \in R^{n}$ are the joint angular displacement, angular velocity, and angular acceleration vectors of the robot, respectively. $M_{0}(\theta) \in R^{n \times n}$ is the nominal symmetric positive definite inertia matrix vector of the robot. $G_{0}(\theta, \dot{\theta}) \in R^{n}$ is the nominal centripetal force and Coriolis force matrix vector. $V_{0}(\theta) \in R^{n}$ is the nominal scale gravity matrix vector. $\tau \in R^{n}$ is the actuator control torque vector. $\delta \in R^{n}$ is the total disturbance of the robot system comprising external disturbance, friction torque, and unmodeled dynamics, which is the uncertainty of system model parameters. $\eta(\theta, \dot{\theta})$ is the uncertainty of system model parameters.

The control objective shows that the control torque $\tau$ is designed to realize the precise position tracking of the robot joint considering the parameter perturbation $\eta(\theta, \dot{\theta})$ and external disturbance $\delta$ in the system.

\subsection{Design of adaptive fuzzy observer}

The fuzzy system has the property of approaching any function. Thus, the dynamic estimation of system total disturbance is realized by fuzzy observation.

Assuming that the desired joint trajectory of the robot is $\theta_{d}$, and the position tracking error is defined as $e=\theta-\theta_{d}$.

The feedback control is applied for the determined part of the system as shown in Eq. (2).

$$
\tau_{1}=\tau-M_{0}(\theta) \ddot{\theta}+G_{0}(\theta, \dot{\theta}) \dot{\theta}+V_{0}(\theta)
$$

Then, the model of Eq. (1) can be expressed as follows:

$M_{0}(\theta) \ddot{e}+G_{0}(\theta, \dot{\theta}) \dot{e}+d=\tau_{1}$ where $d=\eta(\theta, \dot{\theta})+\delta$ is the total disturbance of the system. The fuzzy observer is employed for online estimation and compensation for the total disturbance $d$, and the expression of the fuzzy observer is as follows:

$$
d=\hat{d}+\varepsilon_{d}=\hat{W}_{d}^{*} \varphi_{d}+\varepsilon_{d}
$$

where $\varphi_{d}$ is the fuzzy basis function, $\hat{W}_{d}^{*}$ is the optimal weight of fuzzy system estimation, and $\varepsilon_{d}$ is the approximation error.

Eq. (5) can be obtained by substituting $d$ into Eq. (3) as follows:

$$
M_{0}(q) \ddot{e}+G_{0}(q, \dot{q}) \dot{e}+\hat{W}_{d}^{*} \varphi_{d}+\varepsilon_{d}=\tau_{1}
$$

The sliding mode function is designed as follows:

$s=\dot{e}+c \dot{e}$

where $c>0$, and Eq. (6) satisfies the Hurwitz condition.

Defining new error state variables are $\theta_{1}=e$ and $\theta_{2}=s$, then the following equations can be as follows:

$\left\{\begin{array}{l}\dot{\theta}_{1}=\theta_{2}-c \theta_{1} \\ M_{0} \dot{\theta}_{2}=-G_{0} \theta_{2}+M_{0} c \dot{e}+G_{0} c e-\hat{W}_{d}^{*} \varphi_{d}-\varepsilon_{d}+\tau_{1}\end{array}\right.$

New error state variables $\theta_{1}=e$ and $\theta_{2}=s$ are used as the input of the fuzzy system. The five fuzzy sets are designed for each robot joint, which can form 25 fuzzy rules. The design steps of fuzzy observer are as follows:

(1) Construction of fuzzy system

For the variable $\theta_{i}(i=1,2)$, a fuzzy set $A_{i}^{l_{i}}\left(l_{i}=1,2,3,4,5\right)$ is defined by $p_{i}(i=1,2)$, and a fuzzy system is constructed by using $\prod_{i=1}^{n} p_{i}=p_{1} \times p_{2}=25$ fuzzy rules. Then, the $j$-th fuzzy rule is as follows: $R^{(j)}$ shows that if $x_{1}$ is $\mathrm{A}_{1}^{l_{1}}$ and $x_{2}$ is $\mathrm{A}_{1}^{l_{2}}$, then $\hat{d}$ is $\mathrm{B}^{l_{1} l_{2}}$.

(2) Calculation of fuzzy basis functions

The product inference engine is employed to realize the antecedent reasoning of rules, and the output of the fuzzy system is obtained by solving the average fuzzer as follows:

$$
\hat{d}(\theta / \beta)=\frac{\sum_{l_{i=1}=1}^{5} \sum_{h=1}^{5} \bar{y}_{f}^{h_{2}}\left(\prod_{i=1}^{2} \mu_{A^{4}}\left(\theta_{i}\right)\right)}{\left.\sum_{h_{h}=1 h_{2}=1}^{5} \sum_{i=1}^{5}\left(\prod_{A^{i}}^{2} \mu_{i}\right)\right)}
$$

where $\mu_{A_{i}^{i}}\left(\theta_{i}\right)$ is the membership function of $\theta_{i}$, and $\bar{y}_{f}^{l_{1} l_{2}}$ is a free parameter. Then, fuzzy base vector $\varphi_{d}$ is introduced, and the output of the fuzzy system can be expressed as follows:

$$
\hat{d}=\hat{W}_{d}^{*} \varphi_{d}
$$

where $\varphi_{d}$ is a 25-dimensional fuzzy base vector, in which the $l_{1} l_{2}$-th element is be expressed as follows: 


$$
\varphi_{d l_{1} l_{2}}(\theta)=\frac{\prod_{i=1}^{2} \mu_{A_{i}^{l_{i}}}\left(\theta_{i}\right)}{\sum_{l_{1}=1}^{5} \sum_{l_{2}=1}^{5}\left(\prod_{i=1}^{2} \mu_{A_{i}^{i_{i}}}\left(\theta_{i}\right)\right)}
$$

In the fuzzy observer designed by the above methods, each input variable designs the five membership functions for the two-joint robot, the total number of rules is $5^{2 \times 2}=625$, and many fuzzy rules will lead to excessive calculation. Therefore, the parameters $\hat{W}_{d}$ of fuzzy rules re adaptively designed $\dot{\hat{W}}_{d}^{*}=-\beta \theta_{2} \varphi_{d}^{T}$ to reduce their number, and the expression of the adaptive fuzzy observer is as follows:

$$
\left\{\begin{array}{l}
\hat{d}=\hat{W}_{d}^{*} \varphi_{d} \\
\dot{\hat{W}}_{d}^{*}=-\beta \theta_{2} \varphi_{d}^{T}
\end{array}\right.
$$

\subsection{Robust controller design based on fuzzy observation} If the approximation error $\varepsilon_{d}$ is regarded as the external disturbance of the system, and a criterion signal is defined as $z=s$, then the gain $L_{2}$ of the system is $J=\sup _{\left\|\varepsilon_{d}\right\| \neq 0} \frac{\|z\|_{2}}{\left\|\varepsilon_{d}\right\|_{2}}$.

Theorem: For the error system Eq. (7), if the system adopts the control law Eq. (12) and the adaptive law Eq. (13), and the evaluation function takes $z=s$, then the closed-loop system satisfies $J \leq \lambda$, and $\lambda>0$ is a constant. The following equations can be obtained:

$$
\begin{aligned}
& \tau_{1}=-M_{0} c \dot{e}-G_{0} c e-\frac{1}{2 \lambda^{2}} \theta_{2}+\hat{W}_{d} \varphi_{d}-\frac{1}{2} \theta_{2} \\
& \dot{\hat{W}}_{d}^{*}=-\beta \theta_{2} \varphi_{d}^{T}
\end{aligned}
$$

where $\hat{W}_{d}^{*}$ is the optimal estimation value of the fuzzy system weight $W_{d}$, its error is $\tilde{W}_{d}=\hat{W}_{d}-\hat{W}_{d}^{*}$, and $\beta$ is the adaptive updating coefficient of the weight.

Proof. Lyapunov function is defined as $V=\frac{1}{2} \theta_{2}^{T} M_{0} \theta_{2}+\frac{1}{2 \beta} \operatorname{tr}\left(\tilde{W}_{d}^{T} \tilde{W}_{d}\right)$, in which $\operatorname{tr}(*)$ is expressed as matrix trace $*$.

The derivation of Lyapunov function along the error dynamic Eq. (7) is expressed as follows:

$$
\begin{aligned}
& \dot{V}=\theta_{2}^{T} M_{0} \dot{\theta}_{2}+\frac{1}{2} \theta_{2}^{T} \dot{M}_{0} \theta_{2}+\frac{1}{\beta} \operatorname{tr}\left(\dot{\tilde{W}}_{d}^{T} \tilde{W}_{d}\right) \\
& =\theta_{2}^{T}\left(-G_{0} \theta_{2}+M_{0} c \dot{e}+G_{0} c e-W_{d}^{*} \varphi_{d}-\varepsilon_{d}+\tau_{1}\right)+ \\
& \frac{1}{2} \theta_{2}^{T} \dot{M}_{0} \theta_{2}+\frac{1}{\beta} \operatorname{tr}\left(\dot{\tilde{W}}_{d}^{T} \tilde{W}_{d}\right)=\theta_{2}^{T}\left(-G_{0} \theta_{2}-W_{d}^{*} \varphi_{d}\right. \\
& \left.-\varepsilon_{d}-\frac{1}{2 \lambda^{2}} \theta_{2}+\hat{W}_{d} \varphi_{d}-\frac{1}{2} \theta_{2}\right)+\frac{1}{2} \theta_{2}^{T} \dot{M}_{0} \theta_{2}+ \\
& \frac{1}{\beta} \operatorname{tr}\left(\dot{\tilde{W}}_{d}^{T} \tilde{W}_{d}\right)=\theta_{2}^{T}\left(-\varepsilon_{d}-\frac{1}{2 \lambda^{2}} \theta_{2}+\tilde{W}_{d} \varphi_{d}-\frac{1}{2} \theta_{2}\right)+ \\
& \frac{1}{2} \theta_{2}^{T}\left(\dot{M}_{0}-2 G_{0}\right) \theta_{2}+\frac{1}{\beta} \operatorname{tr}\left(\dot{\tilde{W}}_{d}^{T} \tilde{W}_{d}\right)=-\theta_{2}^{T} \varepsilon_{d}- \\
& \frac{1}{2 \lambda^{2}} \theta_{2}^{T} \theta_{2}+\theta_{2}^{T} \tilde{W}_{d} \varphi_{d}-\frac{1}{2} \theta_{2}^{T} \theta_{2}+\frac{1}{\beta} \operatorname{tr}\left(\dot{\tilde{W}}_{d}^{T} \tilde{W}_{d}\right)
\end{aligned}
$$

The $L$ is defined as follows:

$$
L=\dot{V}-\frac{1}{2} \beta^{2}\left\|\varepsilon_{d}\right\|^{2}+\frac{1}{2}\|z\|^{2}
$$

Then, the following equations can be obtained:

$$
\begin{aligned}
L=- & \theta_{2}^{T} \varepsilon_{d}-\frac{1}{2 \lambda^{2}} \theta_{2}^{T} \theta_{2}+\theta_{2}^{T} \tilde{W}_{d} \varphi_{d}-\frac{1}{2} \theta_{2}^{T} \theta_{2}+ \\
& \frac{1}{\beta} \operatorname{tr}\left(\dot{\tilde{W}}_{d}^{T} \tilde{W}_{d}\right)-\frac{1}{2} \beta^{2}\left\|\varepsilon_{d}\right\|^{2}+\frac{1}{2}\|z\|^{2}
\end{aligned}
$$

where $-\theta_{2}^{T} \varepsilon_{d}-\frac{1}{2 \lambda^{2}} \theta_{2}^{T} \theta_{2}-\frac{1}{2} \beta^{2}\left\|\varepsilon_{d}\right\|^{2}=-\frac{1}{2}\left\|\frac{1}{\lambda} \theta_{2}+\lambda \varepsilon_{d}\right\|^{2} \leq 0$, $\theta_{2}^{T} \tilde{W}_{d} \varphi_{d}+\frac{1}{\beta} \operatorname{tr}\left(\dot{\tilde{W}}_{d}^{T} \tilde{W}_{d}\right)=0$, and $-\frac{1}{2} \theta_{2}^{T} \theta_{2}+\frac{1}{2}\|z\|^{2}=0$, thus $L \leq 0$. The definition of function $L$ shows that $\dot{V} \leq \frac{1}{2} \beta^{2}\left\|\varepsilon_{d}\right\|^{2}-\frac{1}{2}\|z\|^{2}$. According to the Hamilton-Jacobi inequality theorem, if the $L_{2}$ gain's $J$ of the closed-loop system Eq. (7) is less than or equal to the given value $\lambda$, the angular displacement error $e$ and angular velocity error $\dot{e}$ of the robot meet the requirements of $L_{2}$ gain robust convergence.

\section{Results of analysis and discussion}

Taking the dual-joint multi-DOF robot as an example, the proposed adaptive fuzzy observation is compared with Takagi-Sugeno fuzzy observation to verify the effectiveness of the proposed method. The parameters of the two-joint multi-DOF robot are expressed as follows:

$$
\begin{aligned}
& M_{0}(q)=\left[\begin{array}{cc}
1+2.5 \cos q_{2} & 1+\sin x_{2} \\
1+\sin x_{2} & 1
\end{array}\right] \\
& G_{0}(q)=\left[\begin{array}{cc}
-1.5 \dot{q}_{2} \sin q_{2} & -1.5\left(\dot{q}_{1}+\dot{q}_{2}\right) \sin q_{2} \\
-1.5 q_{1} \sin q_{2} & 0
\end{array}\right]^{T} \\
& V_{0}(q)=\left[\begin{array}{c}
2.5 \cos q_{2}+\cos \left(q_{1}+q_{2}\right) \\
\cos \left(q_{1}+q_{2}\right)
\end{array}\right]^{T} \\
& d=\left[\begin{array}{c}
30 \operatorname{sgn} q_{2} \\
30 \operatorname{sgn} q_{4}
\end{array}\right]^{T}
\end{aligned}
$$

The expected command is set as $q_{1 d}=q_{2 d}=\sin t$, and the initial positions are all zero. The parameters of the sliding surface are taken as $c=20$, the fuzzy weight is adaptively updated $\beta=800$, and the $L_{2}$ gain is given indicator $\lambda=0.03$.

\subsection{Tracking performance comparison of systems without interference}

Figs. 2, 3, and 4 respectively show the position tracking error, velocity tracking error, and control input curve of PD compensation control, traditional sliding mode control, and the proposed method. Figs. 2(a), 3(a), and 4(a) can accurately realize position tracking without interference, and the three methods reveal a considerable difference in starting performance. PD compensation control has a large position overshoot (approximately 0.1 rad), and sliding mode control is approximately $0.03 \mathrm{rad}$. The position tracking accuracy of the proposed method is 10 and 3.3 times higher than that of PD compensation and sliding mode controls, respectively, and its position overshoot is approximately 0.01 rad, especially when the position tracking accuracy of two joints 
reaches $5 \times 10^{-5} \mathrm{rad}$. The proposed method is suitable for high-precision tracking occasions. Figs. 2(b), 3(b), and 4(b) show that the sliding mode control and the proposed method have a faster dynamic response than PD compensation control. Figs. 2(c), 3(c), and 4(c) show that sliding mode and $\mathrm{PD}$ compensation controls require a large control input, that is, a large amount of control energy is necessary to achieve the position tracking effect, which is consistent with the proposed method. Simultaneously, the sliding mode control has substantial control torque fluctuation, which is difficult to be applied in practice. The control input torque of the proposed method is relatively smooth, and certain fluctuations are found only during the start-up phase. After stabilization, the amplitude of the proposed method is within $10 \mathrm{~nm}$, which is $1 / 5$ and $1 / 40$ of PD compensation and sliding mode controls, respectively. Therefore, the proposed method has good dynamic and steady-state performance.
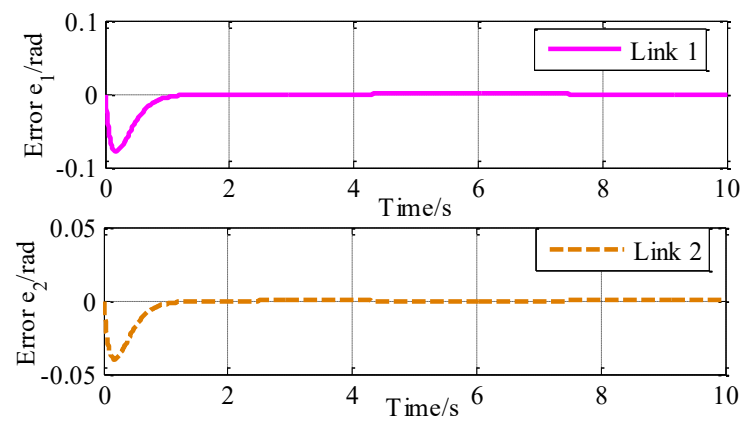

(a) Joint position tracking error
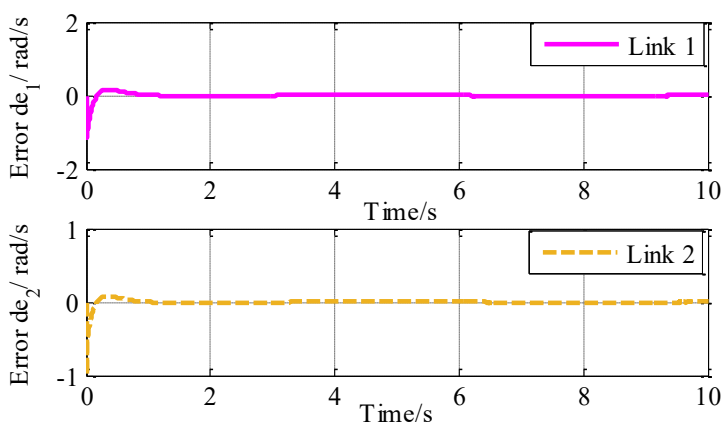

(b) Joint velocity tracking error
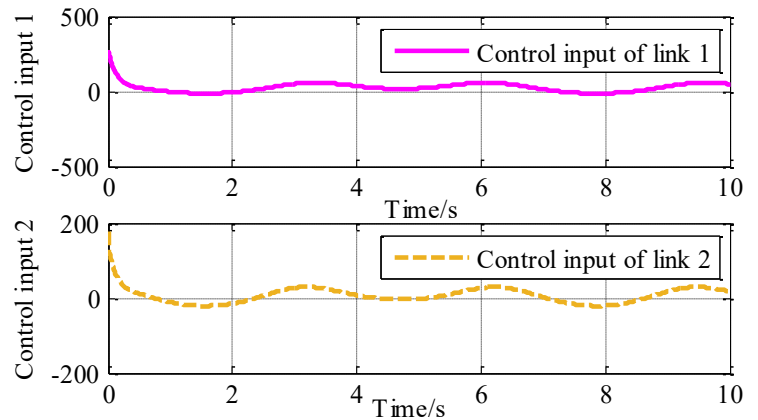

(c) Control input

Fig. 2. Performance curve of PD controlled robot without disturbance

\subsection{Comparison of system tracking performance with interference}

Fig. 5 shows the position tracking error, velocity tracking error, and control input curve of the traditional sliding mode control and the proposed method with friction moment disturbance.
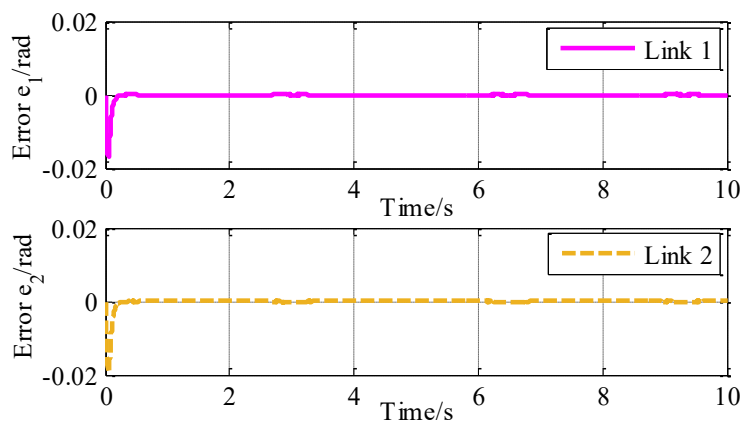

(a) Joint position tracking error
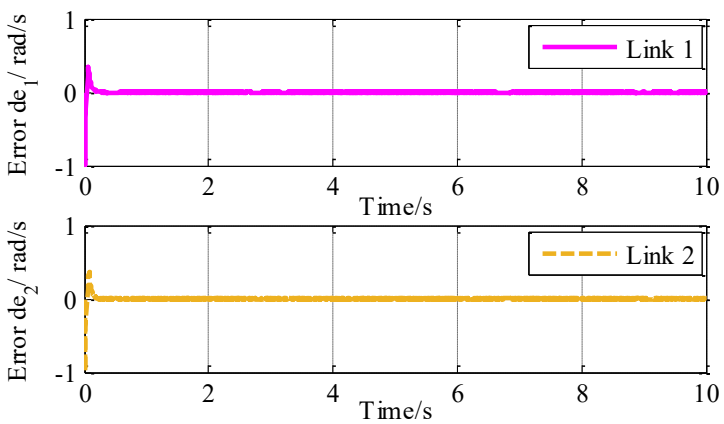

(b) Joint velocity tracking error
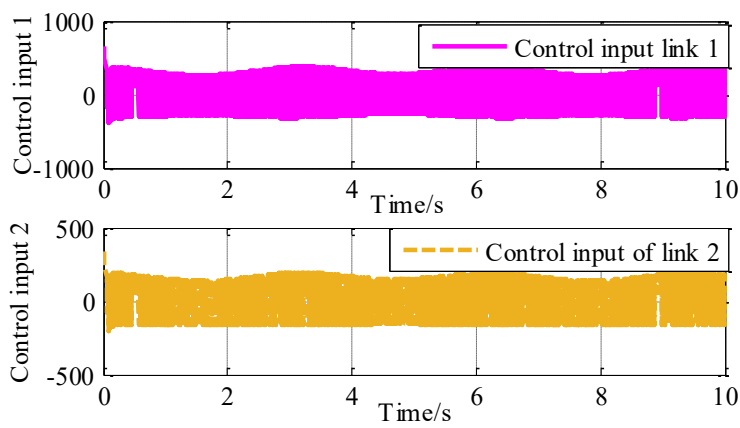

(c) Control input

Fig. 3. Performance curve of sliding mode control robot without disturbance
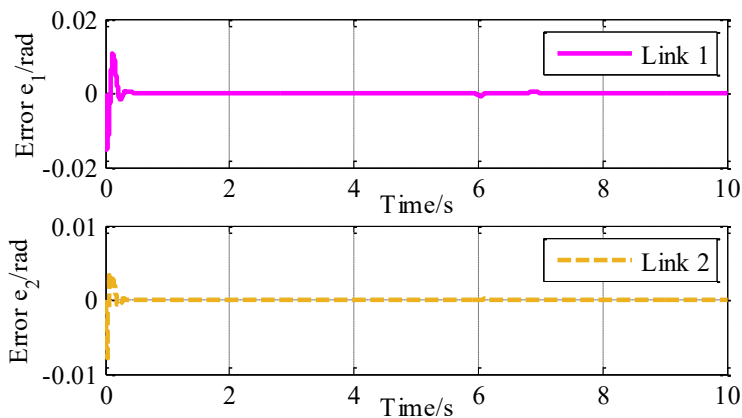

(a) Joint position tracking error
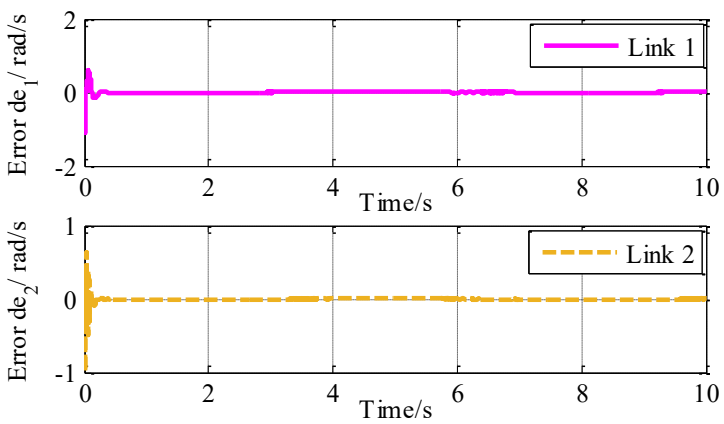

(b) Joint velocity tracking error 

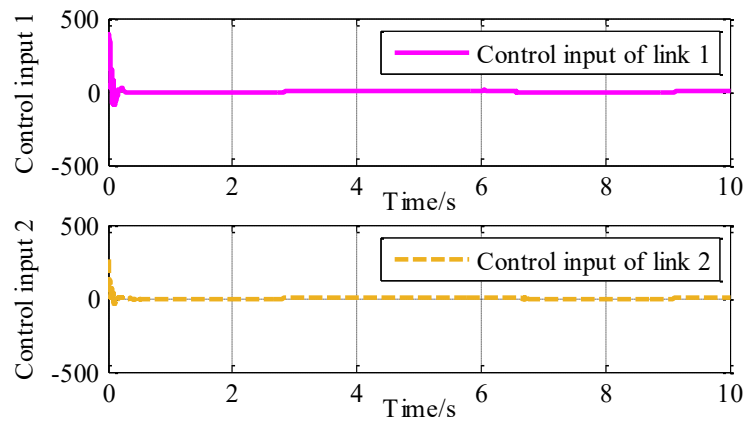

(c) Control input

Fig. 4. Performance curve of the proposed method robot without interference

Fig. 5 reveals that when friction torque disturbance is present, the following conclusions can be drawn. (1) The position tracking error of joints 1 and joint 2 with traditional sliding mode control considerably fluctuates, and the maximum error reaches $0.125 \mathrm{rad}$ and $0.1155 \mathrm{rad}$, respectively. (2) The maximum position tracking error of joints 1 and 2 using the proposed adaptive fuzzy compensation method is $6 \times 10^{-3} \mathrm{rad}$ and $9.5 \times 10^{-3} \mathrm{rad}$, respectively, and the position tracking error is reduced to $1 / 20$ and $1 / 11$ in the traditional sliding mode control, respectively. (3) The speed tracking error of joints 1 and joint 2 using the proposed adaptive fuzzy compensation method remarkably fluctuates in the start-up stage (joints 1 and 2 reach $1 \mathrm{rad} / \mathrm{s}$ ), and the maximum error is $0.07 \mathrm{rad} / \mathrm{s}$ after $0.15 \mathrm{~s}$ stabilization. (4) Joints 1 and 2 using the traditional sliding mode control method are $0.6 \mathrm{rad} / \mathrm{s}$ and $0.65 \mathrm{rad} / \mathrm{s}$, respectively, after $0.15 \mathrm{~s}$ stabilization, and its speed tracking errors are reduced to 8.57 and 9.57 times of the proposed method. Therefore, the proposed method has high accuracy of position and velocity tracking.
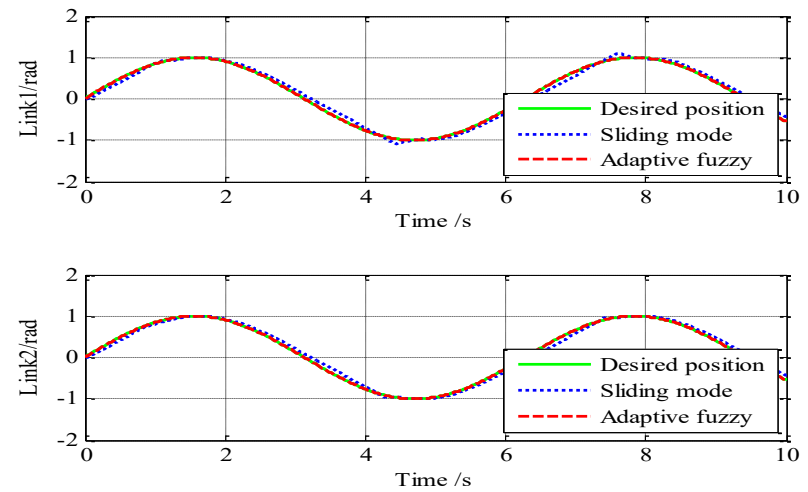

(a) Joint position tracking
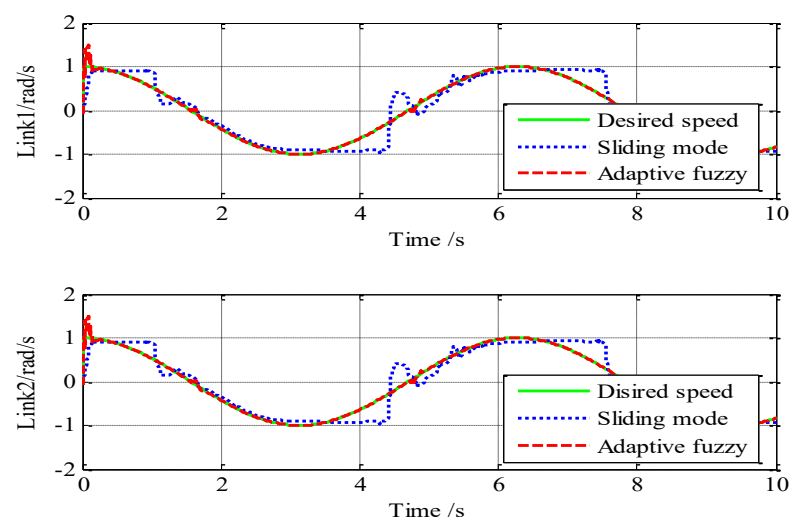

(b) Joint velocity tracking
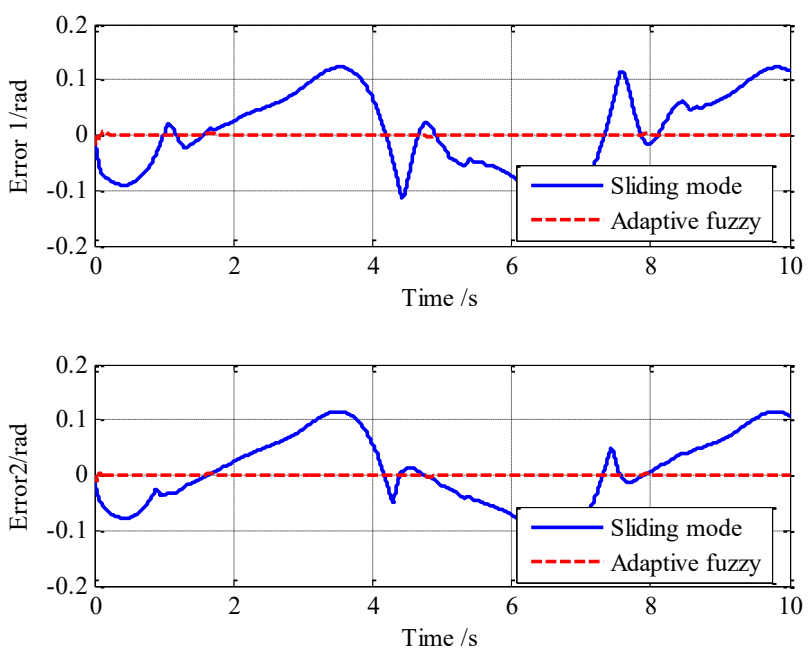

(c) Robot position tracking error
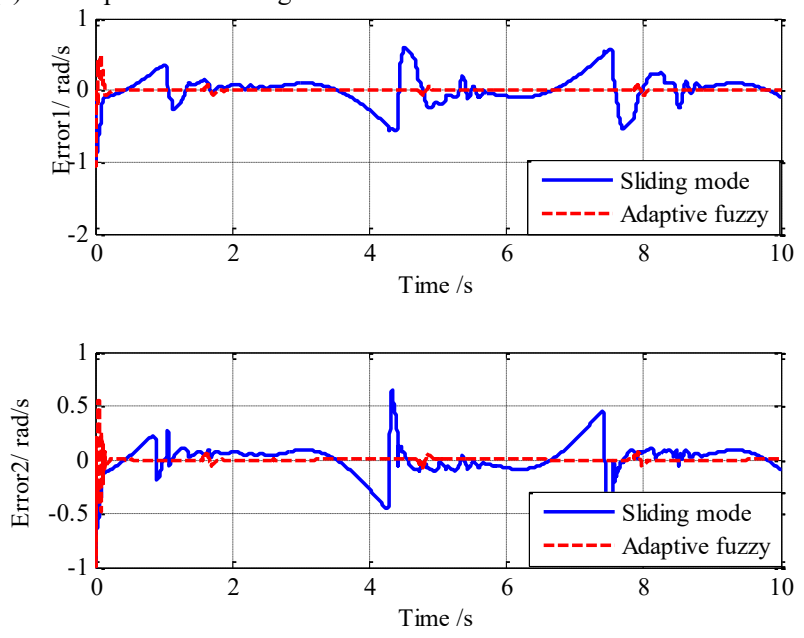

(d) Joint velocity tracking error

Fig. 5. Robot position tracking and error response curve

Figs. 6 and 7 show the robot control input and disturbance estimation curves, respectively. Fig. 6 indicates that the control input of the proposed method is smooth. The overshoot is considerably small when the speed zero point is switched, while the traditional fuzzy estimation has a large overshoot. Fig. 7 shows that the proposed method can accurately estimate the interference, while the estimation accuracy of traditional fuzzy estimation is considerably affected by the number of fuzzy rules. Increasing the number of fuzzy rules (i.e., to increase the number of membership functions) is necessary to improve accuracy. The two-joint robot has two input variables for each joint (i.e., $\theta_{1}=e$, and $\theta_{2}=s$ ), and each input variable corresponds to five membership functions and 625 fuzzy rules. If the membership function becomes seven, then the number of rules is 2401. An excessive number of fuzzy rules will lead to excessive calculation, reduce the response capability of the system, and increase the complexity of the system, which is the purpose of the proposed method. 

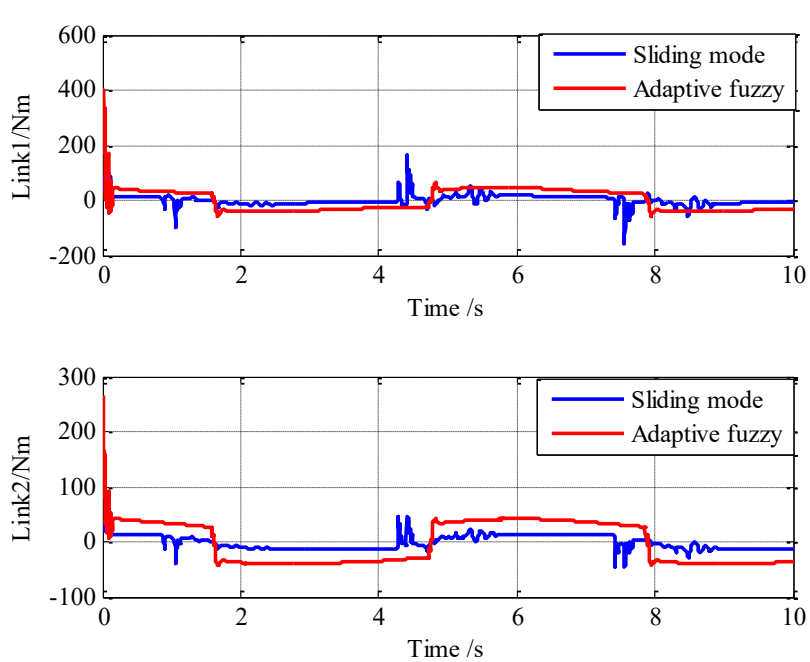

Fig. 6. Robot control input
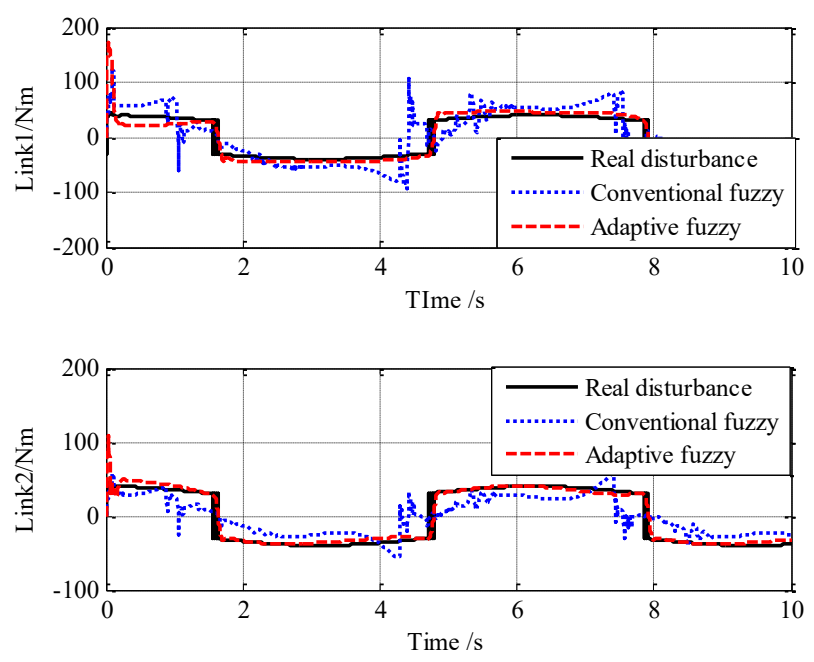

Fig.7. Robot interference estimation

\section{Conclusions}

This study analyzed the internal relationship of the system based on the model characteristics of the robot system to overcome the influence of model parameter change and external disturbance on the tracking accuracy in multijoint robot systems. Moreover, the study examined the tracking and robust performances of the system combining fuzzy and adaptive control theories. The following conclusions could be drawn as follows.

(1) The self-adaptive fuzzy disturbance observer can realize the time-varying system model parameters and the real-time estimation of external disturbance. This observer also has higher accuracy and fewer fuzzy rules than the conventional fuzzy observer.

(2) Compared with the conventional sliding mode control method, the robust control method with neural network estimation compensation can improve the position and velocity tracking accuracy of robot joints by 20 and 9.57 times, respectively.

(3) The output of neural network compensation robust control is smoother than the traditional sliding mode control, and the control energy is small.

This study combines simulation experiments with theories, and the proposed method effectively improves the influence of uncertain factors, such as the change in multijoint robot system model parameters and external disturbance. The control method is simple in structure, convenient for physical implementation, and suitable for the actual working conditions, thus providing a certain guiding significance for the later application in high-performance robot processing.

\section{Acknowledgements}

This work was supported by the Key Scientific Study Projects of Higher Education Institutions of Henan Province (Grant Nos. 20B470003 and 18B470007) and the Promotion Special Project of Scientific Study Program of Henan Province (Grant Nos. 202102210298, 202102210134 and 202102210084).

This is an Open Access article distributed under the terms of the Creative Commons Attribution License

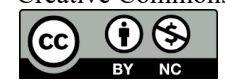

\section{References}

1. Wang, Z.X., Zhang, X.Y., Li, F.F., Zhang, S.Q., Qin, X.S., "Review on the research developments of robot machining systems and cutting chatter behaviors". Journal of Vibration and Shock, 36(14), 2017, pp.147-155, 188.

2. Yi, G.T., Zhu, Z,H., Gong, H., Lu, Z.S., Xiong, H.S., Liu, L., He, W., "Flexible punching system using industrial robots for automotive panels". China Mechanical Engineering, 30(4), 2019, pp.494-497, 503.

3. Wang, R.G., Chen, H.Q., Dai, J.S., "Dynamic stability study of a novel controllable metamorphic palletizing robot mechanism". Journal of Mechanical Engineering, 53(13), 2017, pp.39-47.

4. Tian, W., Jiao, J.S., Li, B., Cui, G.Y., "High precision robot operation equipment and technology in aerospace manufacturing". Journal of Nanjing University of Aeronautics and Astronautics, 52(3), 2020, pp.341-352.

5. Wang, G.X., Cui, Z.Y., "Design of precision pore control system for robot based on lidar". Laser Journal, 40(10), 2019, pp.103-106.

6. Feng, Y.B., Zhao, X.H., He, Z., Li, Z.G., Wang, Y.B., "Depth hover control of spherical robot for internal detection of oil-immersed transformer". Control and Decision, 35(2), 2020, pp.375-381.

7. Zhou, B.H., Wu, Q., "Balancing and optimization of robotic assemble lines with tool and space constraint". Journal of Jilin University (Engineering and Technology Edition), 49(6), 2019, pp.2069-2075.
8. Meng, Z.J., Xu, X.D., Cai, J., Huang, P.F., "Master-slave bilateral adaptive internal model control for space teleoperation robot". Computer Measurement and Control, 19(10), 2011, pp.2424-2426, 2429.Computer Measurement and Control, 19(10), 2011, pp.24242426, 2429.

9. Huynh, B.P., Wu C.W., Kuo, Y.L., "Force/position hybrid control for a Hexa robot using gradient descent iterative learning control algorithm". IEEE Access, (7), 2019 pp.72329-72342.

10. Ma, Y.T., Zheng, R., Yu, C., "Autonomous underwater vehicle deepening control based on transiting target value nonlinear PID". Control Theory and Applications, 35(8), 2018, pp.1120-1125.

11. Zheng, K.M., Zhang, Q.J., "Fuzzy PID control of delta robot based on elastic dynamic model and genetic algorithm". Computer Integrated Manufacturing Systems, 22(7), 2016, pp.1707-1716.

12. Huang, X.R., Song, Y.Y., Li, Q.S., Xiao, X., "A vibration suppression algorithm for industrial robot joint servo system based on internal model control". Transactions of China Electrotechnical Society, 34(3), 2019, pp.497-505.

13. Jiao, J., Jiang, C.H., Jin, R.C., Xu, Z.R., Liu, B., “Adaptive Internal Model Control for Agricultural Robot Steering System". Transactions of the Chinese Society for Agricultural Machinery, 42(10), 2011, 186-191, 234.

14. Liu, L.Y., "Design and application of internal model control for jointed cloud robot with delay time". Journal of Shijiazhuang University of Applied Technology, 31(4), 2019, pp.8-12. 
15. Luc, L.T., Alin, A.S., "Robust adaptive tracking control based on state feedback controller with integrator terms for elastic joint robots with uncertain parameters". IEEE Transactions on Control Systems Technology, 26(6), 2018, pp.2259-2267.

16. Baek, J., Cho, S., Han, S., "Practical time-delay control with adaptive gains for trajectory tracking of robot manipulators". IEEE Transactions on Industrial Electronics, 65(7), 2018, pp.5682-5692.

17. Rubio, Jesus, J.D., "Sliding mode control of robotic arms with deadzone". Institution of Engineering and Technology Control Theory and Applications, 11(8), 2017, pp.1214-1221.

18. Baek, J., Jin, M., Han, S., "A new adaptive sliding-mode control scheme for application to robot manipulators". IEEE Transactions on Industrial Electronics, 63(6), 2016, pp.3628-3637.

19. Nair, R.R., Karki, H., Shukla, A., Behera, L., Jamshidi, M., "Faulttolerant formation control of nonholonomic robots using fast adaptive gain nonsingular terminal sliding mode control". IEEE Systems Journal, 13(1), 2019, pp.1006-1017.

20. Worthmann, K., Mehrez, M.W., Zanon. M., Mann, G.K.I., Gosine, R.G., Diehl, M., "Model predictive control of nonholonomic mobile robots without stabilizing constraints and costs". IEEE Transactions on Control Systems Technology, 24(4), 2016, pp.1394-1406.

21. Incremona, G.P., Ferrara, A., Magni, L., "MPC for robot manipulators with integral sliding modes generation". IEEE/ASME Transactions on Mechatronics, 22(3), 2017, pp.1299-1307.
22. Wang, S.X., Ren, C., Ma, S.G., "Active disturbance rejection control with friction compensation for an omnidirectional mobile robot". Control Theory and Applications, 34(10), 2017, pp.12851292.

23. Zhang, J.H., Cai, C., Liu, X., Zhang, M.L., "Manipulator collision strategy based on second-order feedforward external force observer". Computer Integrated Manufacturing Systems, 25(7), 2019, pp.1775-1783.

24. Chen, E.Z., Chang, J., Li, B., Zhang, G.W., Liu, C., "Control for pitch motion of underwater gliding snake-like robot based on disturbance observer". Journal of Xi'an Jiaotong University, 54(1), 2020, pp.184-192.

25. Li, Z.J., Ye, J.H., Wu, H.B., "Robot collision detection with convolution torque observer and friction compensation". Journal of Zhejiang University (Engineering Science), 53(3), 2019, pp.427434.

26. Huang, G.Y., "Terminal sliding mode control based on neural network disturbance observer". Journal of Jilin University (Engineering and Technology Edition), 41(6), 2011, pp.726-730.

27. Li, C.Y., Wang, W., "Almost disturbance decoupling for dynamic uncertain nonlinear systems via fuzzy output feedback". Control and Decision, 29(5), 2014, pp.779-786.

28. Wu, C.C., Song A., Zhang, H.T., Feng, C., "A backstepping control strategy for prosthetic hand based on fuzzy observation of stiffness". Robot, 35(6), 2013, pp.686-691. 